\title{
BIM CURRICULO: LEVANTAMENTO NOS CURSOS DE ENGENHARIA CIVIL E ARQUITETURA DAS UNIVERSIDADES PÚBLICAS BRASILEIRAS
}

Renan Dinis Pergher-renanpergher@gmail.com

UFPR, Programa de Pós Graduação em Engenharia da Construção Civil

Centro Politécnico

81530-000 - Curitiba - Paraná

Maria do Carmo Duarte Freitas-mcf@ufpr.br

UFPR-Departamento de Ciência e Gestão da Informação

Av. Prefeito Lothário Meissner, 632

80210-170 - Curitiba - Paraná

Resumo: A presença do Building Information Modeling (BIM) enquanto componente curricular nos cursos de Arquitetura e Engenharia Cívil é um tópico em constante desenvolvimento no Brasil. Se por um lado a pesquisa científica tem se expandido e amadurecido, aproximando a academia do tema, por outro o ensino BIM permanece distante da maior parte das graduações. Esta pesquisa realizou um levantamento nas matrizes curriculares (MC) e nos Projetos Políticos Pedagógicos (PPPs) das Instituições de Ensino Superior (IES) públicas do Brasil para verificar a presença ou ausência da Modelagem da Informação da Construção como componente da formação profissional. Foram analisados estatisticamente 171 cursos em 116 instituições, apresentando os resultados em gráficos e mapas. Foi possível concluir a predominante ausência do tema, bem como uma concentração na região sudeste de currículos BIM. Diante disso, se reforçam as necessidades de disseminação do conceito na academia, como meio de consolidação de uma estratégia nacional.

Palavras-chave: BIM Curriculo. Ensino BIM. Graduação AEC.

\section{INTRODUÇÃO}

A história do Building Information Modeling no Brasil é oriunda do começo dos anos 2000, com a adoção da metodologia em escritórios de engenharia e na pesquisa científica (BROCARDO e SHEER, 2017). Um mapeamento se inicia nos anos de 2009 e 2010, com um modesto numero de escritórios de arquitetura e engenharia implementando ou se preparando para realizar uma mudança tecnológica (BARISON, 2015). Com pouquíssimo treinamento disponível e voltados prioritariamente aos softwares, empresas de São Paulo, Rio de Janeiro, Curitiba e Belo Horizonte cautelosamente apostavam nas vantagens do modelo da informação para o aumento de produtividade e qualidade nos serviços.

Em um estudo feito Checcucci, Pereira e Amorin (2011) demonstrou que em 2011, em meio aos participantes do Encontro de Tecnologia da Informação e Comunicação na Construção, a adoção do paradigma BIM ainda estava em fase inicial mesmo nas empresas consideradas "de ponta". Já em 2014, Delatorre e Santos (2014), identificam o reconhecimento, por parte da grande indústria, do papel estratégico do uso da Modelagem da Informação da Construção se refletindo em licitações e tomadas de preço dos grandes contratantes nacionais. 
Como consequência, em 2016 se iniciam os trabalhos para elaboração de uma Estratégia BIM BR, por meio de ações de entidades de classe, empresariado e governo, culminando em 2018 com o lançamento da plataforma BIM BR e assinatura do decreto presidencial $\mathrm{n}^{\circ}$ 9377/2018 (MAGALHÃES,2019). Contudo, apesar do extenso período de adoção e das diversas inciativas públicas e privadas, o estágio brasileiro é inicial (MAGALHÃES, 2019) com apenas 7,5\% das empresas do setor reportando uso de BIM, enquanto 21,7\% desconhecem o uso da metodologia (CASTELO, MARCELLINI e VIANA, 2018). A ausência de profissionais qualificados a transformar esse cenário tem pressionado a academia no sentido de tomar ações de incorporação e disseminação do conhecimento, tanto campo da pesquisa e do ensino.

\section{DISSEMINAÇÃO ACADÊMICA DO BIM NO BRASIL}

Tratar da disseminação do BIM nos ambiente universitários é, em certa medida, compreender as influências da pesquisa científicas nas atividades de ensino. Um retrato disso é a observação que Barison (2015) faz ao notar as instituições pioneiras na educação BIM UFBA, UFF, UPM, USP, UNICAMP e UFPR - também eram, em 2010, os expoentes na pesquisa. Se isso já foi condição para presença do BIM em sala de aula, com as diretrizes curriculares das engenharias de 2019, a estratégia BIM BR de 2018 e a constante exigência do mercado, a atualização curricular das graduações em Arquitetura, Engenharia e Construção (AEC) tal disposição apresenta um conflito. Contudo, demonstraremos a sinergia entre pesquisa e ensino e sua evolução no Brasil.

\subsection{Pesquisa em BIM}

O primeiro levantamento da pesquisa sobre BIM no Brasil realizado por Andrade e Ruchel (2009) onde, para os autores, se revelou uma insipiência em um tema pouco estudado e publicado em apenas 13 artigos. Em 2011, Checcucci, Pereira e Amorim encontram um estado mais profícuo da pesquisa, com um total de 44 publicações entre 2005 à 2010. Os autores destacavam que: (a) os pesquisadores concentravam-se nas regiões Sul e Sudeste do país e, portanto, era necessária a criação ou ampliação de redes de pesquisas; (b) já se identificava a preocupação da academia em inserir BIM no ensino, com 9 artigos tratando de experiências já realizadas; (c) havia pouco material de acesso livre sobre BIM, com algumas teses e dissertações não publicadas no banco de teses da Capes nem no Portal Domínio Públicos e os anais de alguns importantes eventos científicos não disponíveis na internet (CHECCUCCI, PEREIRA E AMORIM, 2011). 
Figura 1 - Distribuição Territorial da titulação acadêmica sobre BIM baseado em Machado, Ruschel e Scheer, 2017.

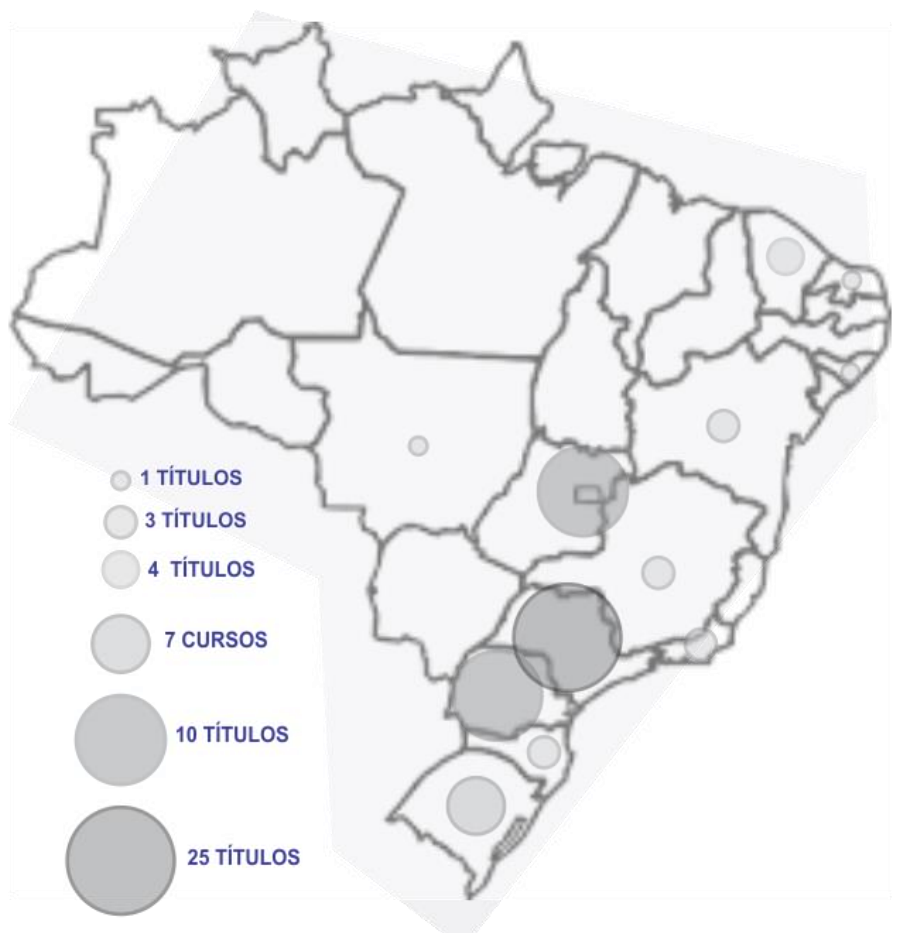

Fonte: O autor, 2020.

Em 2017 Machado, Ruschel e Scheer resgatam o avanço na pesquisa BIM, levantando 405 trabalhos publicados dentre os quais 75 são produtos de titulações em programas de pós graduação, demonstrando o amadurecimento científico nacional. A regionalização se expande, com a criação de grupos de pesquisa em múltiplas instituições, mas sem superar as constatações de Checcucci, Pereira e Amorim (2011), conforme apresentado na "Figura 1". Um numero expressivo de participações em eventos com artigos publicados também revelou um maior engajamento isolado de pesquisadores, com pouquíssima colaboração entre instituições nacionais e internacionais (MACHADO, RUSCHEL E SCHEER, 2017). Associado a isso, Checcucci (2019) explicita 12 pesquisas de mestrado e doutorado voltadas ao ensino e aprendizagem de BIM. Nesses estudos, as reflexões sobre os componentes curriculares é constante, revelando a preocupação da associação da pesquisa consolidada em BIM à formação dos Arquitetos e Engenheiros.

\subsection{Ensino de BIM}

O rebatimento da pesquisa nos cursos de graduação em AEC possui alguma expressões desde o principio da adoção do conceito. Em 2010, Menezes et. Al. discutem os conteúdos curriculares e as teorias de projeto arquitetônico, apontando para a incompatibilidade desses com o potencial das recentes TICs, incluindo o BIM. Para os autores a estrutura acadêmica hegemônica é um entrave na formatação de profissionais arquitetos capazes pois o BIM e o "projeto simultâneo" ou colaborativo fomentam ainda mais o descolamento entre tais teorias vigentes e a prática do planejamento da edificação (MENEZES et. al, 2010). Com isso, Benedetto, Bernarde e Pires (2016) afirmam que para se inserir efetivamente BIM aos currículos várias definições devem ser feitas, e citam: Possíveis dificuldades, Nível de formação a ser ofertado, organização do currículo, $\mathrm{O}$ método de ensino-aprendizagem a ser incorporado, Atividades propostas e mecanismos de avaliação (BENEDETTO, BERNARDE 
\& PIRES, 2016). Alguns outros fatores menos específicos são citadas pelos autores como capacidade de investimento, planejamento das etapas, tempo, recursos didáticos e capacitação docente. Aguiar e Pereira (2017) reforçam a desatualização curricular e da mentalidade escolar nas faculdades de engenharia, negando o avanço das TICs para difusão e o engrandecimento do conhecimento um ato colaborativo.

Em 2018 foi constituída a iniciativa do Encontro Nacional de ensino de BIM (ENEBIM), onde se percebeu uma diversidade de propostas qualificadas para a formação (CHECCUCCI, 2019). Durante os eventos foi possível constatar: que: (a)As experiências acontecem a partir de um esforço pessoal dos docentes envolvidos com o componente curricular, que se articulam com colegas para viabilizar as disciplinas; (b) acontecem de forma pontual nos currículos dos cursos; (c) continuam abrangendo as fases iniciais do ciclo de vida da edificação. Também ficou clara a demanda por docentes capacitados em BIM para dar conta do desafio de formação existente no Brasil (CHECCUCCI, 2019). Para autora, o reconhecimento estatístico do panorama nacional era uma tarefa importante, realizando assim seu resgate das pesquisas sobre o ensino, já mencionado. Na mesma linha, este trabalho focará agora nos componentes curriculares como índice de disseminação.

\section{LEVANTAMENTO CURRICULAR DAS IES PÚBLICAS BRASILEIRAS}

Para levantar a disseminação do conceito BIM no ambiente acadêmico foi proposta a leitura das matrizes curriculares dos cursos de graduação em Arquitetura e Urbanismo e Engenharia Civil. A busca foi por disciplinas que contivessem em seu título todos os sinônimos para Modelagem da Informação da Construção, bem como nomes genéricos que remetessem à computação, informática e tecnologia aplicada na construção civil ("Quadro 1"), sendo considerados apenas os que contivessem em suas ementas o conteúdo BIM. Foram consideradas todas as disciplinas afirmativas ao tema, tanto da grade curricular obrigatória quanto das disciplinas optativas, independente sua periodicidade.

Quadro 1 - Título das Disciplinas Verificadas em Ementa

\begin{tabular}{|c|c|c|}
\hline Informática aplicada a Arquitetura & $\begin{array}{c}\text { Tecnologia da Informação e } \\
\text { Comunicação }\end{array}$ & $\begin{array}{c}\text { Modelagem } \\
\text { Computacional }\end{array}$ \\
\hline $\begin{array}{c}\text { Tecnologia Computacional para } \\
\text { Engenharia }\end{array}$ & $\begin{array}{c}\text { Projeto Auxiliado por } \\
\text { Computador }\end{array}$ & $\begin{array}{c}\text { Tecnologia } \\
\text { Computacional na } \\
\text { Construção Civil }\end{array}$ \\
\hline Modelagem Arquitetônica & Engenharia Computacional & Programação do Projeto \\
\hline
\end{tabular}

Como unidade de análise, delimitou-se ás instituições de ensino superior públicas. Se justifica esse recorte para tornar tangível o levantamento de dados, verificar a sintonia de política institucional para BIM nas autarquias públicas e pelo papel destacado que essas ainda cumprem na formação profissional no Brasil. Com isso, se encontrou na base de dados do MEC (2020) 205 IES de administração pública, nas três esferas do poder (Federal, Estadual e Municipal). Dessas, apenas 115 possuem cursos de Arquitetura, ou Engenharia Civil ou ambos, distribuídos conforme Gráfico 1. 


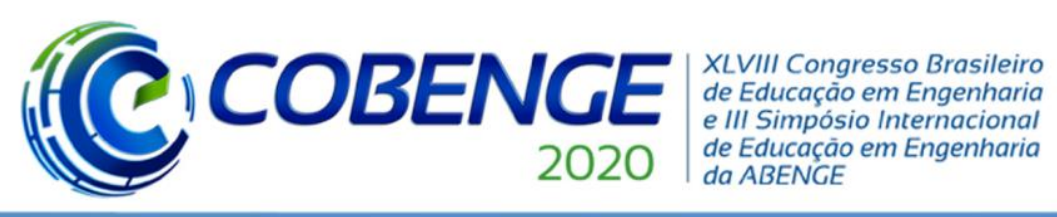

"Os desafios para formar hoje o engenheiro do amanhã"

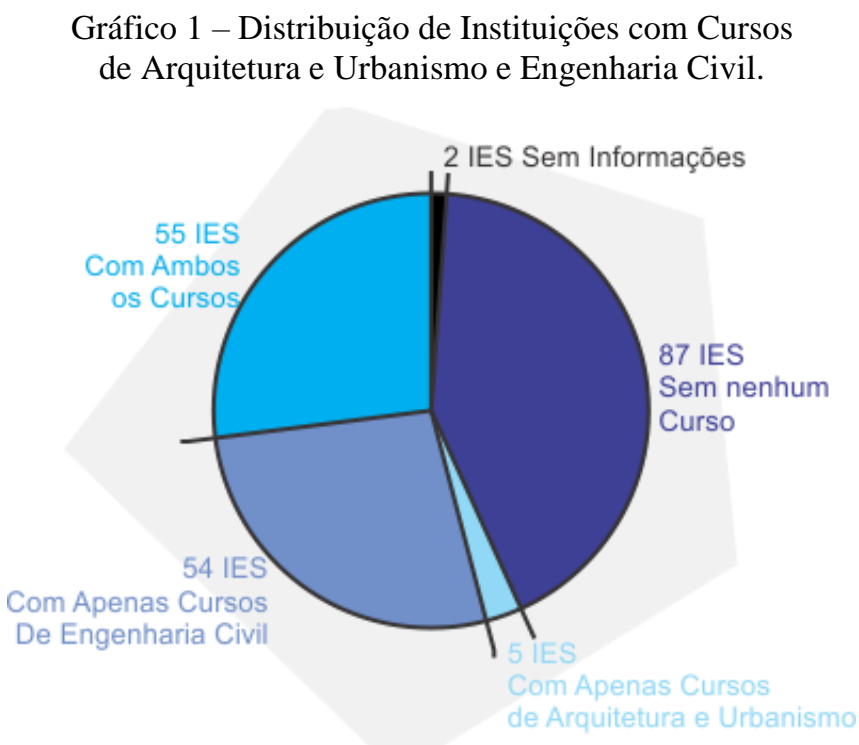

Fonte: O autor, 2020.

Em casos de instituições multi campi, com mais de um modelo curricular, foi considerado o projeto pedagógico comum, prevalecendo o resultado positivo caso fosse encontrado em pelo menos em uma versão. Como fonte primaria foi utilizado os sites institucionais e seus acervos online, buscando nas paginas de curso, pró-reitorias de graduação, setores ou departamentos a grade curricular atualizada e o Projeto Político Pedagógico dos cursos, para análise das ementas.

Gráfico 2 - Percentual de disponibilidade de Materiais Curriculares e Pedagógicos dos Cursos em Site.

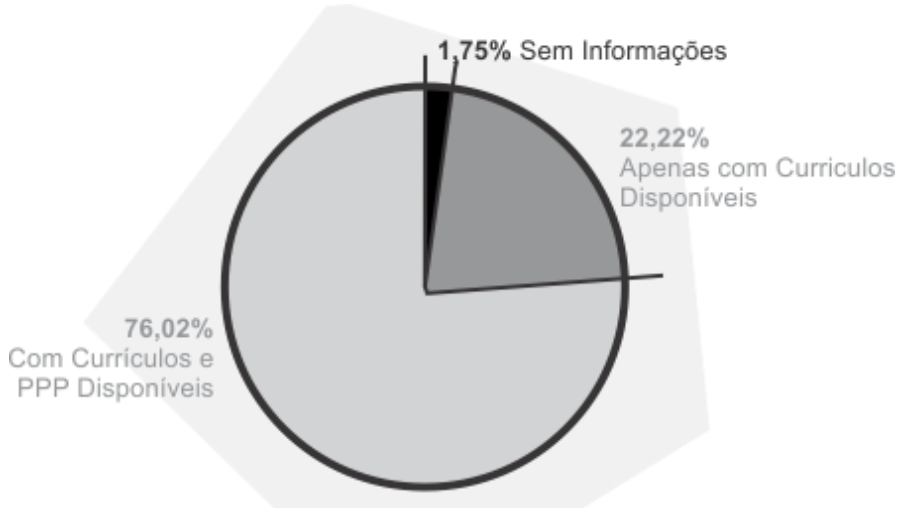

Fonte: O autor, 2020.

Quando não disponível em site, distribuição expressa no "Gráfico 2", fora feito contato direto com as coordenações de curso e chefias de departamento para obtenção de dados de fonte secundaria, solicitando esclarecimentos e o envio dos materiais. De 41 e-mail enviados, apenas 1 ofereceu retorno. 
(C) COBENGE 2020

"Os desafios para formar hoje o engenheiro do amanhã"

\subsection{Resultados}

A análise de dados revelou que, do total de 171 currículos estudados, apenas $21,05 \%$ possui em algum grau a menção à BIM. Desse modo, se identificou que $25 \%$ dos cursos de arquitetura contemplavam o conteúdo, enquanto apenas 18,91\% dos cursos de Engenharia Civil apresentaram resultados afirmativos. Em verdade, mais da metade dos cursos, 55\% para Arquitetura e 65\% para Engenharia Civil não possui nenhuma indicação da presença do tema na grade curricular conforme "Gráfico 4" e "Gráfico 5".

Gráfico 4 - Resultados Encontrados Para os Cursos de Arquitetura e Urbanismo.

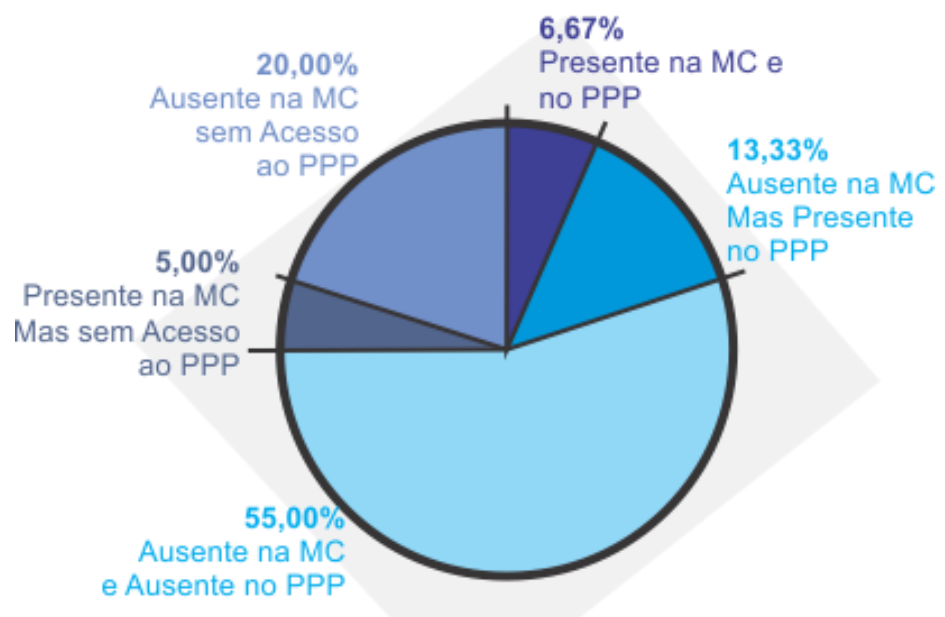

Fonte: O autor, 2020.

Gráfico 5 Resultados Encontrados Para os Cursos de Engenharia Civil.

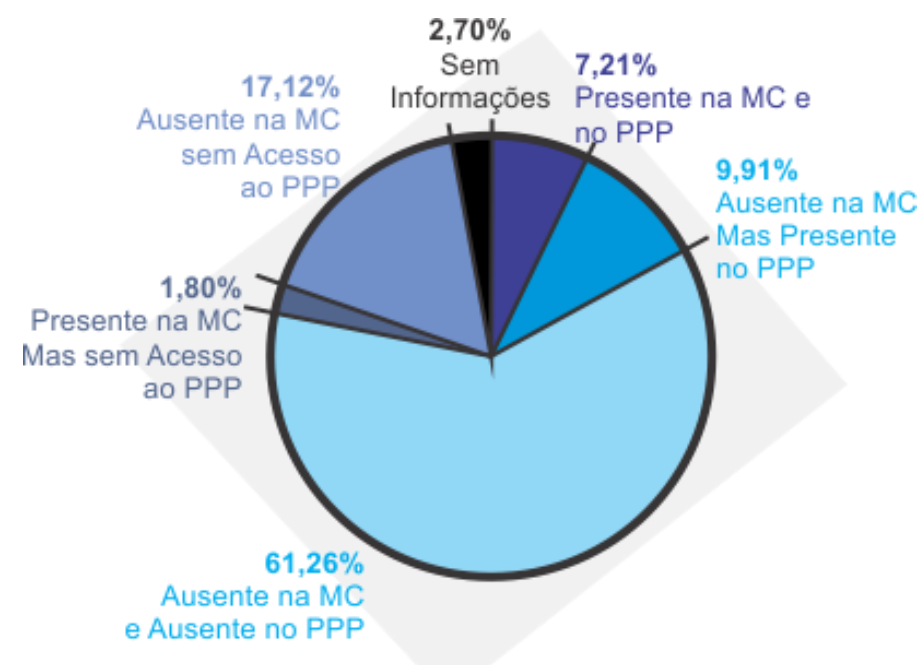

Fonte: O autor, 2020.

A distribuição territorial também foi destacada na "Figura 2", demonstrando uma concentração desses cursos na região sudeste (50\% do total de currículos estudados). Apenas o estado de Minas Gerais representa 11,34\%, sendo um resultado afirmativo em 11 dos 23 cursos, $50 \%$ nos cursos engenharia civil e 42,85\% nos de Arquitetura e Urbanismo no estado. 


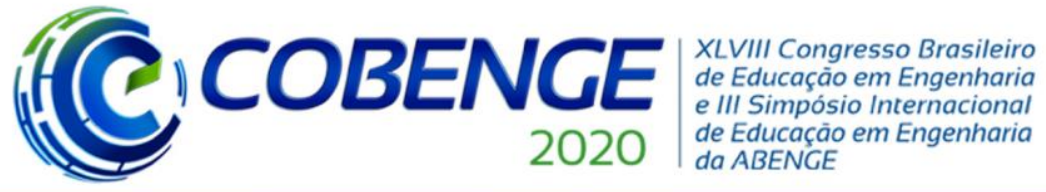

"Os desafios para formar hoje o engenheiro do amanhã"

Doze unidades federativas brasileiras não apontaram nenhuma IES com nenhum vestígio de currículo BIM.

Figura 2 - Distribuição Territorial das IES com cursos com Curriculo BIM.

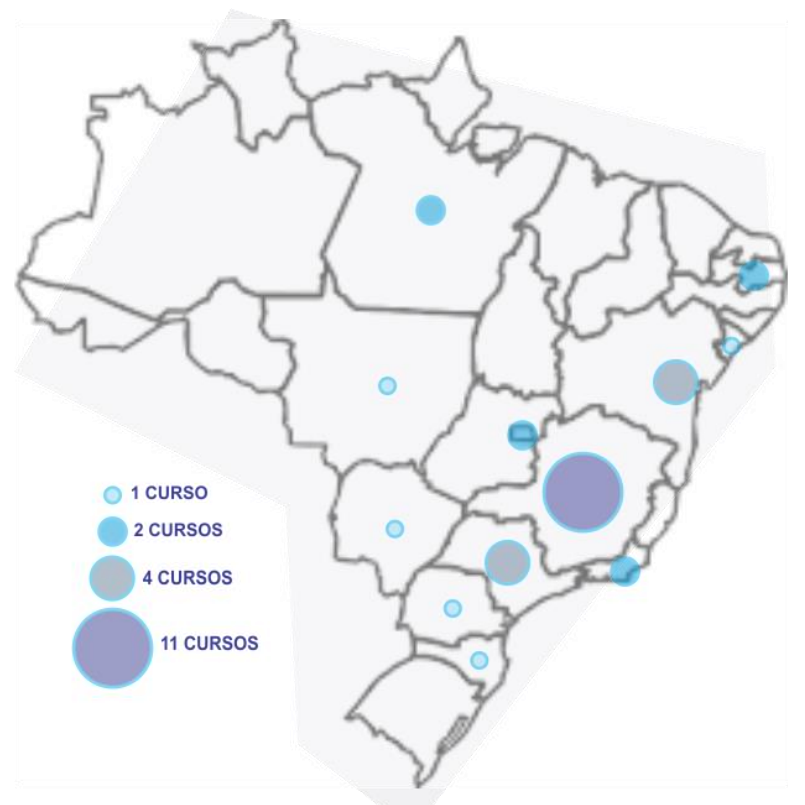

Fonte: O autor, 2020.

\subsection{Discussão}

Há alguns fatores a serem considerados antes de realizar apontamentos sobre as informações levantadas. Primeiro sobre as diretrizes curriculares nacionais para ambos os cursos onde no caso da Arquitetura e Urbanismo, essas foram estabelecidas em 2010 pouco antes do aumento significativo de publicações científicas sobre BIM do periodo entre 2011 e 2015 (MACHADO; RUSCHEL; SCHEER, 2017). No caso da Engenharia Civil estas foram reformuladas em 2019, estimulando a implementação das TICs na graduação, que resulta em reformulações curriculares em andamento, 25 casos levantados por esta pesquisa. Segundo é a distribuição desigual de IES públicas e cursos em AEC no território onde o estado do Acre possui apenas um curso de Engenharia Civil e nenhum de Arquitetura enquanto São Paulo e Minas Gerais possuem um total de 24 e 23 cursos, respectivamente, representando somados $27,48 \%$ dos cursos nacionalmente.

Posto isso, é notória a concentração de currículos que abordem BIM na região sudeste brasileira e a limitada aparição dos estados do norte. Pode-se associar isso o volume de atividades econômicas e a participação no PIB que cada estado e região tem: Sudeste com $52,9 \%$ e o Norte com 16,5\% (IBGE, 2019). Também, os dados expressam uma relação entre a presença curricular e os levantamentos da pesquisa científica do tema onde, seja pela existência de docentes competentes ou linhas de pesquisa institucionalizadas, há uma corroboração produção de publicações e a existência de disciplinas. Chama-se ainda a atenção para o crescimento de 6 instituições em 2010 (BARISON, 2015) para as 116 encontradas hoje apontando para um avanço no setor público na elaboração de currículos BIM. 


\section{CONSIDERAÇÕES FINAIS}

O presente trabalho buscou atualizar os dados sobre o ensino de BIM em AEC, iniciando pelo setor público. Para que se monte um quadro completo é fundamental que o mesmo levantamento seja feito nos mais de 1500 cursos reconhecidos pelo MEC. Também, revisitar o tema quando passado o período de adaptação curricular para as engenharias deverá fornecer indicativos mais fieis sobre o esforço das IES públicas em aproximar-se dos currículos BIM.

Contudo, não se deve crer que presença curricular reflete em ensino BIM de sucesso. Para afirmar isso é fundamental o uso de métricas como as sugeridas por Succar (2010) ou frameworks como o de Barison (2015).

De todo modo, os desafios da disseminação da filosofia BIM e do curriculo BIM permanecem, no Brasil e no Mundo. Avançar na capacitação dos Docentes, na aquisição de tecnologias e nos estudos de caso são fundamentais para atendermos a meta de um Brasil BIM em 2021 e as necessidades da Industria 4.0 na Construção Civil.

\section{Agradecimentos}

Agradecimento a equipe do GPCIT, laboratório de gestão de informação da UFPR, ao PPGECC e seus professores e demais estrutura da Universidade Federal do Paraná. Também a CAPES, CNPQ e a Fundação Araucária - Governo do Estado do Paraná, pelo apoio e financiamento da pesquisa.

\section{REFERÊNCIAS}

AGUILAR, M; PEREIRA, M. Colaboração em BIM: Uma Experiência de Aprendizado em um Curso de Engenharia Civil. SIMPÓSIO BRASILEIRO DE GESTÃO E ECONOMIA DA CONSTRUÇÃO, 3. Fortaleza, 2017

ANDRADE, M. L. V. X; RUSCHEL, R. C. BIM: conceitos, cenários das pesquisas publicadas no Brasil e tendências. In: SIMPÓSIO BRASILEIRO DE QUALIDADE DE PROJETOS, 1., 2009, São Carlos. Anais [...]. São Carlos: RiMa, 2009.

BARISON, M.B. Introdução de Modelagem da Informação da Construção (BIM) no Currículo - uma contribuição para formação do projetista. Tese de Doutorado. Universidade de São Paulo. São Paulo, 2015.

BENEDETTO, H.; BERNARDES, M; PIRES, R. W. Ensino de BIM no Brasil: Análise do Cenário Acadêmico. INFORMÁTICA NA EDUCAÇÃO: teoria \& prática Porto Alegre, v.20, n.2, Mai./Ago. 2017.

BRASIL. Decreto n. 9.377, de 17 de maio de 2018. Institui a Estratégia de Disseminação do Building Information Modelling. Diário Oficial da União, Brasília, Edição 95, Seção 1, p. 3, mai. 2018. Atos do Poder Executivo. Disponível em: http://www.planalto.gov.br/ccivil_03/_ato2015-2018/2018/Decreto/D9377.htm. Acesso em: 5 ago. 2020

BROCARDO, F.L. M; SCHEER, S. O Uso Da Modelagem Da Informação Da Construção 4d (Bim 4d) Em Projetos De Obras Militares. Anais [do] 1o Simpósio Brasileiro de Tecnologia de Informação e Comunicação na Construção (SBTIC 2017) Fortaleza, CE: Marketing Aumentado, 2017 ISBN: 978-85-67169-10-1 (PDF) acesso em 05 Ago. 2020 
CASTELO, Ana Maria; MARCELLINI, Laura; VIANA, Iuri. A Construção Digital parte2. Disponível em: <https://blogdoibre.fgv.br/posts/construcao-digital-parte-2> Acesso em 05 ago. 2020

CHECCUCCI, E. S.; Teses e Dissertações Brasileiras sobre BIM: Uma Análise do Período de 2013 a 2018. PARC Pesquisa em Arquitetura e Construção, Campinas, SP, v. 10, p. e019008, 2019, ISSN 1980-6809. Disponível em: <

https://periodicos.sbu.unicamp.br/ojs/index.php/parc/issue/view/1563/showToc>. Acesso em: 05 ago. 2020

CHECCUCCI, E. S.; PEREIRA, A. P. C.; AMORIM, A. L. A Difusão das Tecnologias BIM por Pesquisadores do Brasil. In: ENCONTRO NACIONAL DE TECNOLOGIA DE INFORMAÇÃO E COMUNICAÇÃO NA CONSTRUÇÃO CIVIL, 15., Salvador, 2011. Anais [...]. Salvador: LCAD/PPGAU - UFBA, 2011.

DELATORRE, J. P.; SANTOS, E. T. Introdução de Novas Tecnologias: O caso do BIM em Empresas da Construção Civil. In: ENCONTRO NACIONAL DE TECNOLOGIA DO AMBIENTE CONSTRUÍDO, 5., Maceió, 2014.

IBGE. Dados Econômicos, 2019. Disponível em: <www.ibge.gov.br>. Acesso em: 05 Ago. 2020

MACHADO, F.; RUSCHEL, R. C.; SCHEER, S. Análise da produção científica brasileira sobre a Modelagem da Informação da Construção. Ambient. constr. [online]. Porto Alegre, v. 17, n. 4, p. 359-384, out. /dez. 2017. DOI: http://dx.doi.org/10.1590/s1678-86212. Acesso em: 05 Ago. 2020

MAGALHÃES, C.R. Panorama BIM: Visões e Reflexões da Adoção pela Indústria da Construção Civil Brasileira. Anais [do] 2o Simpósio Brasileiro de Tecnologia de Informação e Comunicação na Construção (SBTIC 2019) Campinas, SP, 2019. Disponível em: <https://antaceventos.net.br/index.php/sbtic/sbtic2019/paper/viewFile/152/237> acesso em 05 Ago. 2020

MENEZES, A. M. et al. A adequação (ou não) dos aplicativos BIM às teorias contemporâneas de ensino de projeto de edificações. SIGRADI 2010 / Disrupción, modelación y construcción: Diálogos cambiantes. p. 55-57, 2010

MINISTÉRIO DA EDUCAÇÃO (MEC) Cadastro Nacional de Cursos e Instituições do Ensino Superior - E-MEC. Brasília, 2020. Disponível em < https://emec.mec.gov.br/> Acessado em 25 mai 2020

RUSCHEL, R. C. ; ANDRADE, M. L; MORAIS, M. O ensino de BIM no Brasil: onde estamos? Ambiente Construído, Porto Alegre, v. 13, n. 2, p. 151-165, abr./jun. 2013

SUCCAR, B. The five components of BIM performance measurement. In: CIB WORLD BUILDING CONGRESS, 18., 2010, Salford. Proceedings... Salford: CIB, 2010. p. 1-14. 


\title{
BIM CURRICULO: SURVEY IN THE CIVIL ENGINEERING AND ARCHITECTURE COURSES OF BRAZILIAN PUBLIC UNIVERSITIES
}

\begin{abstract}
The presence of Building Information Modeling (BIM) as a curricular component in Architecture and Civil Engineering courses is a topic in constant development in Brazil. If, on the one hand, scientific research has expanded and matured, bringing the academy closer to the theme, on the other hand, BIM teaching remains of most graduations. This research carried out a survey in the curricular matrices (MC) and in the Pedagogical Political Projects (PPPs) of the Public Higher Education Institutions (HEIs) in Brazil to verify the presence or absence of Construction Information Modeling as a component of professional training. There were statistically transit 171 courses in 116 institutions, they choose the results in graphs and maps. It was possible to materialize the predominant theme, as well as a concentration in the southeast region of BIM curricula. Therefore, the need to disseminate the concept in academia is reinforced, as a means of consolidating a national strategy.
\end{abstract}

Keywords: BIM Curriculum, BIM Teaching, AEC Graduations 